\title{
Electrophysiological Characteristics of Obstructive Sleep Apnea Syndrome with Insomnia: Polysomnography and Cardiopulmonary Coupling Analysis
}

\author{
Hea Ree Park, Eun Yeon Joo, Seung Bong Hong \\ Department of Neurology, Neuroscience Center, Samsung Medical Center, Samsung Biomedical Research Institute, Samsung Advanced Insti- \\ tute for Health Sciences and Technology, Sungkyunkwan University School of Medicine, Seoul, Korea
}

Received November 1, 2015 Revised December 28, 2015

Accepted December 29, 2015

Address for correspondence

Eun Yeon Joo, MD, PhD

Department of Neurology,

Neuroscience Center,

Samsung Medical Center,

Samsung Biomedical Research

Institute, Samsung Advanced

Institute for Health Sciences and

Technology, Sungkyunkwan

University School of Medicine,

81 Irwon-ro, Gangnam-gu,

Seoul 06351, Korea

Tel: $+82-2-3410-3597$

Fax: +82-2-3410-0052

E-mail: ejoo@skku.edu
Objectives: To characterize sleep of subjects with obstructive sleep apnea syndrome (OSA) with insomnia compared to OSA without insomnia in terms of polysomnography (PSG) and cardiopulmonary coupling (CPC) analysis. Methods: Subjects with OSA (apnea-hypopnea index, AHI $\geq 5 / h, n=200$ ) were enrolled and divided into subjects OSA with insomnia (OSA-I) and subjects with OSA only (OSA-O). OSA-I complained of difficulty falling and/or staying asleep at an initial interview in clinic. We compared demographics including mood states, daytime sleepiness, PSG, and CPC parameters between groups, and performed correlation analyses between PSG and CPC parameters for each group. Results: Female ratio was higher in OSA-I than OSA-O. OSA-I were older and slimmer than OSA-O. OSA-O were much drowsier (Epworth Sleepiness Scale 10.0 vs. 6.8). However, mood states were not different between two groups. OSA-I showed significantly longer sleep latency and lower sleep efficiency than OSA-O. Despite of higher arousal index (AI) and AHI of OSA-O, wakefulness after sleep onset was greater in OSA-I. There was no significant difference of CPC parameters between two groups after adjustment of AHI. In correlation analyses, low frequency coupling and high frequency coupling duration were associated with $\mathrm{AHI}, \mathrm{AI}$, and lowest $\mathrm{SaO}_{2}$ in both groups. Conclusions: OSA-I demonstrated more fragmented sleep architecture and disruptive sleep in spite of lower sleep-disordered breathing related distress than OSA-O. CPC analysis is unable to differentiate sleep patterns of OSA subjects with or without insomnia. It is needed to explore factors causing fragmented sleep architecture and disruptive sleep rather than respiratory disturbances in OSA subjects complaining of insomnia.

J Sleep Med 2015;12(2):53-58

\section{Introduction}

Obstructive sleep apnea syndrome (OSA) and insomnia are two of most common sleep disorders encountered in the sleep clinic population, and substantial overlap between these two disorders was reported in previous studies. ${ }^{1-5}$ Considering a common concurrence of two disorders, understanding about characteristics of OSA with insomnia patients is important in order to optimize detection and treatment. However, little is known about OSA with insomnia patients' unique characteristics, and clinicians tend to focus only on their respiratory symptoms in diagnosis and treatment.

This is an Open Access article distributed under the terms of the Creative Commons Attribution Non-Commercial License (http://creativecommons.org/licenses/by-nc/3.0) which permits unrestricted non-commercial use, distribution, and reproduction in any medium, provided the original work is properly cited.
Overnight polysomnography (PSG) is the most commonly used test to diagnose sleep disorders, and especially it is gold standard for diagnosis of OSA. On the while, standard PSG is expensive and subjects feel inconvenience due to many electrodes and wires. Cardiopulmonary coupling (CPC) analysis is more convenient and less expensive because it uses a single channel electrocardiography (ECG) recording, and the association between the CPC indices and the sleep quality were proven in both OSA and insomnia in previous studies..$^{6-8}$

The aim of this study is to characterize sleep and sleep quality of subjects with OSA with insomnia compared to OSA without insomnia in terms of PSG and CPC analysis. 


\section{Methods}

\section{Subjects}

This is a retrospective study of OSA subjects who performed PSG in our hospital from November, 2012 to October, 2014. We included the patients who visited not only sleep clinic, but also psychiatric and ENT outpatient clinic. We defined OSA subjects as subjects whose apnea-hypopnea index (AHI) was over $5 / \mathrm{h}$. We excluded subjects who had other sleep disorder (i.e., restless leg syndrome, periodic limb movements during sleep, parasomnia) except chronic insomnia, other neurologic disorder and acute medical illness at the time of PSG, which may affect PSG and CPC indices. Two hundred subjects were finally included in this study. We divided subjects into two groups by their chief complaints at the first visit of our clinic. We designated patients whose chief complaint was insomnia as 'OSA with insomnia' (OSA-I), patents whose main complaint was snoring and stop breathing as 'OSA only' (OSA-O). One examiner reviewed all subjects' medical records, and subjects whose chief complaint was 'insomnia', 'difficulty falling asleep', 'wake up a lot', 'awakening too early', or 'difficulty returning asleep' were classified as OSA-I, and subjects whose chief complaint was 'snoring,' 'stop breathing during sleep', 'apnea' were classified as OSA-O. We only reviewed subjects' chief complaints and did not consider subjects' associated symptoms found during interview or questionnaire to classify subjects. The body mass index (BMI), Beck Anxiety Index (BAI), Beck Depression Index (BDI), Epworth Sleep Scale (ESS) was measured for the subjects in the clinic. All subjects were evaluated with overnight PSG, and we extracted the CPC parameters from single ECG signal from each PSG data to estimate the sleep quality.

\section{Overnight polysomnography}

Sleep studies were performed by Embla N7000\&RemLogic (Embla Systems, Denver, CO, USA). The determination of sleep stage and arousal was measured by electroencephalogram (C3-A2, C4-A1, O1-A2, O2-A1), bilateral electrooculograms and chin electromyogram. Other devices included piezo bands to check chest and abdominal exclusion, finger pulse oximetry, single-lead ECG in the modified V2 lead, bilateral leg electromyograms, pre-tracheal microphone to check snoring, and video monitoring to check body position. Airflow was monitored with thermistors and nasal-cannula pressure transducer during diagnostic sleep studies. Sleep and arousal scoring were performed using standard criteria. The PSG were scored for sleep and sleep-disorders breathing events using the 2007 guidelines of the American Academy of Sleep Medicine. ${ }^{9-11}$ From these measures, amounts of each sleep stage, latency to each sleep stage, total sleep time (TST), sleep efficiency (SE), and wake after sleep onset (WASO) were all calculated.

\section{Cardiopulmonary coupling analysis}

Cardiopulmonary coupling analysis was performed on the single channel ECG data extracted from the diagnostic PSG using available software, RemLogic CPC analyzer. It is composed of a total of five parameters: 1) high-frequency coupling (HFC, spectrogram peaks in the frequency range of 0.1 to 0.5 $\mathrm{Hz}$ ), which indicates stable sleep; 2) low-frequency coupling (LFC, spectrogram peaks in the frequency range of 0.01-0.1 $\mathrm{Hz}$ ), which indicates unstable sleep; 3 ) very-low-frequency coupling (VLFC, spectrogram peaks in the frequency range of $0.00391-0.01 \mathrm{~Hz}$ ), which indicates awake or rapid eye movement sleep; 4) other (spectrogram peaks other than HFC, LFC, and VLFC, typically <1-2\%); and 5) elevated low-frequency coupling (e-LFC, a subset of LFC with especially large low-frequency power), which correlates with sleep fragmentation and sleep apnea. ${ }^{12,13}$

\section{Statistical analysis}

Statistical analyses were performed with SPSS software (SPSS 18.0, SPSS Inc., Chicago, IL, USA). The significance level was set at $p$ value less than 0.05 . Two-sample independent $t$ test was used to assess differences of demographics, PSG and CPC data between the OSA-I group and the OSA-O group. Median regression model was used to compare CPC data between the two groups after correcting the effects of AHI to CPC parameters, and AHI was transformed using natural log due to highly skewed distribution. Partial Spearman correlations between CPC parameters and PSG data in each group were calculated after adjusting for age and sex.

\section{Results}

\section{Demographics}

72 subjects were designated as OSA-I and 128 subjects as OSA-O. Demographic and clinical assessment data for subjects are presented in Table 1. In both groups, the proportion of male was predominant, but ratio of female in the OSA-I group is higher than the OSA-O group (38.9\% in OSA-I vs. $6.8 \%$ in OSA-O, $p<0.001)$. The mean age of OSA-I group is higher than OSA-O group (56.4 \pm 11.2 in OSA-I vs. $44.6 \pm 11.9$ in OSA-O, $p<0.001$ ), and BMI of OSA-I group was lower than OSA-O group (24.4 \pm 3.6 in OSA-I vs. $26.0 \pm 3.4$ in OSA-O, $p=0.003)$. In daytime sleepiness scales, mean ESS values of OSA-I group were higher than OSA-O group $(6.8 \pm 5.1$ in OSA-I vs. $10.0 \pm 4.7$ in OSA-O, $p<0.001$ ). There were no significant differences in BDI and BAI between OSA-I and OSA-O groups. 


\section{PSG and CPC parameters}

Comparison of PSG and CPC parameters of both groups are presented in Table 2. In the PSG comparisons, the OSA-I group showed significantly longer sleep latency $(16.9 \pm 21.1$ vs. $8.3 \pm 8.1, p=0.001)$, lower SE (77.3 \pm 22.4 vs. $85.0 \pm 10.5, p=0.007$ ) and shorter TST ( $341.3 \pm 61.6$ vs. $370.9 \pm 62.6, p=0.001$ ) than OSA-I group. OSA-O group presented significantly higher AHI (21.1 \pm 18.8 vs. $37.6 \pm 23.4, p<0.001)$, higher arousal index

Table 1. Baseline characteristics in patients of OSA with insomnia and OSA only groups

\begin{tabular}{lccc}
\hline & $\begin{array}{c}\text { OSA-I } \\
(\mathrm{n}=72)\end{array}$ & $\begin{array}{c}\text { OSA-O } \\
(\mathrm{n}=128)\end{array}$ & $p$ \\
\hline Male, n (\%) & $44(61.1)$ & $118(92.2)$ & $0.000^{*}$ \\
Age, years & $56.4 \pm 11.2$ & $44.6 \pm 11.9$ & $0.000^{*}$ \\
BMI & $24.4 \pm 3.6$ & $26.0 \pm 3.4$ & $0.003^{*}$ \\
ESS & $6.8 \pm 5.1$ & $10.0 \pm 4.7$ & $0.000^{*}$ \\
BDI & $9.6 \pm 9.9$ & $8.4 \pm 6.4$ & 0.354 \\
BAI & $8.8 \pm 6.1$ & $9.8 \pm 7.0$ & 0.414 \\
\hline
\end{tabular}

${ }^{*} p<0.05$, independent $t$-test. OSA: obstructive sleep apnea syndrome, OSA-I: subjects OSA with insomnia, OSA-O: subjects with OSA only, ESS: Epworth Sleepiness Scale, BDI: Beck Depression Index, BAI: Beck Anxiety Index
(AI) (32.9 \pm 20.0 vs. $26.1 \pm 15.6, p=0.008)$ and decreased lowest $\mathrm{O}_{2}$ saturation (85.7 \pm 5.4 vs. $\left.81.1 \pm 7.5, p<0.001\right)$ than OSA-I group. Although both AHI and AI of OSA-O group were higher than OSA-I group, WASO was greater in OSA-I group.

In the comparison of CPC parameters, only LFC duration was greater in OSA-O than OSA-I ( $45.0 \pm 22.0 \%$ vs. $40.4 \pm 21.5 \%$, $p=0.027$ ), and there was no significant difference in other CPC parameters. After adjustment of AHI using median regression model, differences of all CPC parameters were not significant (Table 3).

\section{Correlation analyses between PSG parameters and CPC parameters}

In OSA-I group, after adjustment of age, gender, and BMI, the correlations between PSG parameters and CPC parameters analyzed by Spearman's partial correlation analysis model, was presented in Table 4 . HFC was negatively correlated with AI, AHI (rho $=-0.477, p<0.001$ and $r h o=-0.464, p<0.001$ ), and did not show significant correlation with TST, sleep latency, $\mathrm{SE}$, lowest $\mathrm{O}_{2}$ saturation, and WASO. LFC and e-LFC was positively correlated with AI, AHI ( $\mathrm{rho}=0.546, p<0.001$ and rho $=0.589, p<0.001$ in LFC, rho $=0.544, p<0.001$ and rho $=0.626$, $p<0.001$ in e-LFC), and showed weak negative correlation with

Table 2. Polysomnography and CPC parameters in patients of OSA with insomnia and OSA only groups

\begin{tabular}{|c|c|c|c|}
\hline & OSA-I $(n=72)$ & OSA-O $(n=128)$ & $p$ \\
\hline \multicolumn{4}{|l|}{ Overnight polysomnography } \\
\hline Time in bed (min) & $454.5 \pm 51.1$ & $435.8 \pm 57.9$ & $0.023^{*}$ \\
\hline Total sleep time (min) & $341.3 \pm 61.6$ & $370.9 \pm 62.6$ & $0.001^{*}$ \\
\hline Sleep latency (min) & $16.9 \pm 21.1$ & $8.3 \pm 8.1$ & $0.001^{*}$ \\
\hline REM latency (min) & $125.9 \pm 82.8$ & $103.0 \pm 71.6$ & 0.051 \\
\hline Sleep efficiency (\%) & $77.3 \pm 22.4$ & $85.0 \pm 10.5$ & $0.007^{*}$ \\
\hline Arousal index $(/ \mathrm{hr})$ & $26.1 \pm 15.6$ & $32.9 \pm 20.0$ & 0.008 \\
\hline N1 (\%) & $23.0 \pm 14.2$ & $23.6 \pm 15.0$ & 0.798 \\
\hline N2 (\%) & $55.0 \pm 13.7$ & $51.9 \pm 12.8$ & 0.107 \\
\hline N3 (\%) & $2.8 \pm 5.5$ & $4.0 \pm 5.8$ & 0.134 \\
\hline REM (\%) & $19.2 \pm 8.0$ & $20.1 \pm 6.2$ & 0.409 \\
\hline Apnea/hypopnea index (/hr) & $21.1 \pm 18.8$ & $37.6 \pm 23.4$ & $0.000^{*}$ \\
\hline WASO (\%) & $21.8 \pm 12.0$ & $13.7 \pm 10.9$ & $0.000^{*}$ \\
\hline Lowest $\mathrm{O}_{2}$ saturation & $85.7 \pm 5.4$ & $81.1 \pm 7.5$ & $0.000^{*}$ \\
\hline \multicolumn{4}{|l|}{ CPC statistics } \\
\hline High frequency coupling duration (\%) & $43.4 \pm 22.1$ & $38.7 \pm 20.7$ & 0.134 \\
\hline Low frequency coupling duration (\%) & $40.4 \pm 21.5$ & $45.0 \pm 22.0$ & $0.027^{*}$ \\
\hline Very low frequency coupling duration (\%) & $15.6 \pm 11.5$ & $13.0 \pm 8.3$ & 0.067 \\
\hline Total e-LFC duration (\%) & $23.5 \pm 21.6$ & $29.2 \pm 23.4$ & 0.087 \\
\hline
\end{tabular}

${ }^{*} p<0.05$, independent t-test. OSA: obstructive sleep apnea syndrome, OSA-I: subjects OSA with insomnia, OSA-O: subjects with OSA only, CPC: cardiopulmonary coupling, REM: rapid eye movement, WASO: wakefulness after sleep onset, e-LFC: elevated low-frequency coupling 
lowest $\mathrm{O}_{2}$ saturation (rho $=-0.299, p=0.012$ and rho $=-0.279$, $p=0.020$ ). LFC and e-LFC did not show significant correlation with TST, sleep latency, SE, and WASO, neither. VLFC did not show correlation with all PSG parameters.

In OSA-O group, after adjustment of age, gender, and BMI, the correlations between PSG parameters and CPC parameters analyzed by Spearman's partial correlation analysis model, was presented in Table 5. HFC was negatively correlated with AI, AHI (rho $=-0.511, p<0.001$ and $\mathrm{rho}=-0.462, p<0.001$ ), and positively correlated with lowest $\mathrm{O}_{2}$ saturation ( $\mathrm{rho}=0.381$, $p<0.001)$. HFC did not show significant correlation with TST, sleep latency, SE, and WASO. LFC and e-LFC were positively correlated with AI, AHI (rho $=0.527, p<0.001$ and rho $=0.531$, $p<0.001$ in LFC, rho $=0.442, p<0.001$ and $\mathrm{rho}=0.432, p<0.001$ in e-LFC) and negatively correlated with lowest $\mathrm{O}_{2}$ saturation (rho $=-0.420, p<0.001)$. LFC and e-LFC did not show significant correlation with TST, sleep latency, SE, and WASO, neither. VLFC showed weak positive correlation with WASO (rho $=0.353, p<0.001)$ and weak negative correlation with TST, $\mathrm{SE}, \mathrm{AHI}(\mathrm{rho}=-0.202, p=0.024$ and $\mathrm{rho}=-0.337, p<0.001$ and rho $=-0.219, p=0.014$, respectively).

\section{Discussion}

Insomnia is one of common complaints of OSA subjects although there is limited understanding about the factors that make differences between OSA subjects complaining daytime sleepiness and subjects complaining difficulty in falling asleep. Several studies insisted that sleepiness and sleeplessness are both end of the spectrum of OSA manifestation and it is related to individual susceptibility, however, it is still unclear what determines this individual difference of susceptibility in OSA. ${ }^{1,14}$ This study aimed to find the determinant factors into two distinct syndromes by comparing available electrophysiological information between OSA subjects who complained insomnia and who did not. Furthermore, we analyzed not only subjects' demographics, mood status, sleep parameters of PSG but also CPC data to find out the characteristics of insomnia-presenting subjects with OSA compared to OSA subjects with insomnia-related symptoms.

In comparisons of demographics, female ratio was significantly higher in OSA-I (38.9\%) than OSA-O (6.8\%). Female OSA patients were revealed to have different clinical manifestation from male patients; they had more frequent insomnia, headache, fatigue, depression, and use of sedatives. ${ }^{15,16} \mathrm{Al}$ though it is not evident whether these differences are related to different pathophysiology of upper airway movements, craniofacial morphology, or to show different baseline personality between genders, female is one of the important factors associated with insomnia-related symptoms in subjects with OSA.
Subjects with OSA-I were older and slimmer than OSA-O. Although there is no report of direct correlation between old age and insomnia-presenting OSA, insomnia-OSA association has been frequently reported amongst elderly patients, and this report is parallel with previous findings. ${ }^{1,17,18}$ Overweight is well-known risk factor of OSA, but subjects with OSA plus insomnia tend to be lighter than OSA only patients in one study. ${ }^{19}$

The PSG findings of OSA-I demonstrated shorter TST, lower SE, longer sleep latency, and higher WASO than OSA-O in spite of their AHI, RDI, and degree of desaturation were lower

Table 3. Comparison of CPC parameters in patients of OSA with insomnia and OSA only groups after adjustment AHI, RDI, lowest $\mathrm{SaO}_{2}$

\begin{tabular}{clllll}
\hline & & HFC & LFC & VLFC & e-LFC \\
\hline OSA with insomnia & t value & 1.66 & -1.29 & 0.99 & 0.13 \\
(reference: OSA only) & $\operatorname{Pr}>|\mathrm{t}|$ & 0.239 & 0.200 & 0.323 & 0.894 \\
\hline
\end{tabular}

CPC: cardiopulmonary coupling, OSA: obstructive sleep apnea syndrome, AHI: apnea/hypopnea index, RDI: respiratory disturbance index, HFC: high frequency coupling, LFC: low frequency coupling, VLFC: very low frequency coupling, e-LFC: elevated low-frequency coupling

Table 4. Partial Spearman correlation analysis between PSG parameters and CPC parameters after adjustment of age, gender, and BMI in patients of OSA with insomnia $(n=72)$

\begin{tabular}{lrcccccc}
\hline & TST & SL & SE & AI & AHI & LowO $_{2}$ & WASO \\
\hline HFC & 0.105 & 0.002 & 0.026 & $-0.477^{\dagger}$ & $-0.464^{\dagger}$ & 0.165 & -0.177 \\
LFC & -0.030 & -0.048 & 0.006 & $0.546^{\dagger}$ & $0.589^{\dagger}$ & $-0.299^{*}$ & 0.090 \\
VLFC & -0.130 & 0.080 & -0.060 & -0.061 & -0.152 & 0.203 & 0.155 \\
e-LFC & 0.028 & -0.067 & 0.042 & $0.544^{\dagger}$ & $0.626^{\dagger}$ & $-0.279^{*}$ & -0.011 \\
\hline
\end{tabular}

Spearman's rho values are presented. ${ }^{*} p<0.05,{ }^{\dagger} p<0.01$. PSG: polysomnography, CPC: cardiopulmonary coupling, BMI: body mass index, OSA: obstructive sleep apnea syndrome, TST: total sleep time, SL: sleep latency, SE: sleep efficiency, AHI: apnea/hypopnea index, $\mathrm{LowO}_{2}$ : lowest $\mathrm{O}_{2}$ saturation, HFC: high frequency coupling, LFC: low frequency coupling, VLFC: very low frequency coupling, e-LFC: elevated low-frequency coupling

Table 5. Partial Spearman correlation analysis between PSG parameters and CPC parameters after adjustment of age, gender, and BMI in patients of OSA only $(n=128)$

\begin{tabular}{lccccccc}
\hline & TST & SL & SE & AI & AHI & LowO $_{2}$ & WASO \\
\hline HFC & 0.061 & -0.101 & 0.130 & $-0.511^{\dagger}$ & $-0.462^{\dagger}$ & $0.381^{\dagger}$ & -0.089 \\
LFC & 0.022 & 0.030 & 0.016 & $0.527^{\dagger}$ & $0.531^{\dagger}$ & $-0.413^{\dagger}$ & -0.060 \\
VLFC & $-0.202^{*}$ & $0.168^{\dagger}$ & -0.337 & -0.108 & $-0.219^{*}$ & 0.108 & $0.353^{\dagger}$ \\
e-LFC & 0.054 & 0.013 & 0.069 & $0.442^{\dagger}$ & $0.432^{\dagger}$ & $-0.420^{\dagger}$ & -0.102 \\
\hline
\end{tabular}

Spearman's rho values are presented. ${ }^{*} p<0.05,{ }^{\dagger} p<0.01$. PSG: polysomnography, CPC: cardiopulmonary coupling, BMI: body mass index, OSA: obstructive sleep apnea syndrome, TST: total sleep time, SL: sleep latency, SE: sleep efficiency, AHI: apnea/hypopnea index, $\mathrm{LowO}_{2}$ : lowest $\mathrm{O}_{2}$ saturation, HFC: high frequency coupling, LFC: low frequency coupling, VLFC: very low frequency coupling, e-LFC: elevated low-frequency coupling 
than OSA-O. This is important finding because lower SE and higher WASO are closely related to poor sleep quality of subjects, although we did not perform the research using questionnaire about sleep quality to these patients. ${ }^{20}$ These PSG characteristics of OSA-I are coincident with previous studies demonstrating that severity of sleep-disordered breathing symptoms of OSA with insomnia patients were similar or milder than OSA without insomnia patients. ${ }^{4,21}$ Contradictory result was reported that Smith et al. found a positive correlation between the presence of insomnia and OSA severity. ${ }^{22}$ These findings implied that severity of sleep-disordered breathings was not sole determinant of sleep quality in OSA-I. Therefore, clinicians should not be focus only on the sleep-disordered breathing symptoms in OSA patients when they mainly complain of sleep onset or maintaining insomnia.

Current study presented that OSA-O showed higher AHI and lower $\mathrm{SaO}_{2}$, which indicate more severe sleep-disordered breathing symptoms than OSA-I. Correlations between sleepdisordered breathing severity and coincident insomnia in OSA patients have been uncertain in previous studies, and the results of our study have two possibilities. First is that patients with insomnia have milder sleep-disordered breathing symptoms, and the other is the presence of selective bias in subjects who had visited to sleep clinic at university-affiliated hospital. If a subject suffers from insomnia, he or she seems more likely to visit sleep clinic even though his sleep-disordered breathing is mild.

In comparison of CPC data between OSA-I and OSA-O, LFC was higher in OSA-O than OSA-I. However, indices related to sleep-disordered breathing such as AHI was significantly higher in OSA-O, and there was no significant difference of LFC between two groups after adjustment of these sleep-disordered breathing indices using regression analysis. Close relationship between CPC parameters and sleep-disordered breathing symptoms of OSA subjects are well known in previous studies. ${ }^{6,7}$ Therefore, difference of LFC between OSAI and OSA-O was probably to reflect the severity difference of sleep-disordered breathing symptoms rather than different characteristics between two groups.

Correlation analysis between PSG and CPC parameters did not show significant differences between OSA-I and OSA-O. In both groups, HFC, LFC, and e-LFC showed significant correlation with $\mathrm{AI}, \mathrm{AHI}$, and lowest $\mathrm{SaO}_{2}$, and these parameters have insignificant correlation with TST, sleep latency, SE, and WASO. It may indicate that CPC parameters mainly suggest features of sleep-disordered breathing even in OSA-I, and they cannot explain the high WASO and low SE in OSA-I. Therefore, it turns out that CPC analysis is limited to differentiate sleep of OSA subjects with or without insomnia. Additionally, we should suspect sleep-disordered breathing even in insom- nia-complaining subjects when they showed low HFC and high LFC in CPC analysis.

It is notable that VLFC was weakly correlated with TST, SE, and WASO only in OSA-O. VLFC generally indicates awakening from sleep or REM sleep, and VLFC of OSA subjects is higher than normal subjects. ${ }^{7}$ Therefore, we presumed that severe OSA patients, mostly included in OSA-O, have severe sleep-disordered breathing related arousals enough to affect TST, SE, and WASO. However, this explanation should be confirmed by subgroup analyses regarding OSA severity in the future.

The limitation of current study is lack of measurement of subjective sleep satisfaction such as subjective TST, sleep latency, and sleep quality index. However, we found significant difference in objective sleep measurements such as sleep latency, TST, WASO, which are closely related to sleep quality. Further research is needed to compare subjective sleep quality between OSA subjects who complained insomnia and who did not.

To summarize, the most important finding of this study is that OSA-I have more fragmented sleep architecture and disruptive sleep than subjects with OSA-O, and sleep-disordered breathing is not the main factor of their poorer sleep. With these PSG and CPC data in subjects with OSA-I, it is not easy to define the relevant features to cause fragmented sleep architecture and disruptive sleep. Insomnia presenting subjects with OSA had more frequent psychiatric diseases such as depression or anxiety. ${ }^{1,4,21,22}$ Nevertheless, we could not find any differences of BDI and BAI between two groups in this study. As noted that physical complaints such as pain, fatigue might affect sleep quality, insomnia patients with OSA reported more frequent physical complaints. ${ }^{1,4,21}$ Absence of information about somatic symptoms might be a limitation of this study. In the future, extensive research is needed to explore the relevant factors of fragmented sleep architecture and disruptive sleep in insomnia-presenting subjects with OSA.

In conclusions, OSA-I demonstrated more fragmented sleep architecture and disruptive sleep regarding PSG parameters in spite of lower sleep-disordered breathing related distress than OSA-O. CPC parameters are unable to differentiate sleep patterns of OSA subjects with or without insomnia. It is needed to explore the factors causing fragmented sleep architecture and disruptive sleep rather than respiratory disturbances during sleep in OSA subjects complaining of insomnia-related symptoms.

\section{Acknowledgments}

This research was supported by Basic Science Research Program through the National Research Foundation of Korea funded by the Ministry of Science, ICT and Future Planning, Republic of Korea (No. 2014R1A1A3049510) and by Samsung Biomedical Research Institute grant (\#OTX0002111). 


\section{REFERENCES}

1. Benetó A, Gomez-Siurana E, Rubio-Sanchez P. Comorbidity between sleep apnea and insomnia. Sleep Med Rev 2009;13:287-293.

2. Benetó A, Cambra M, Gómez E, Rubio P. [Sleep apnea and insomnia: a frequent association]. Med Clin (Barc) 2008;131:756.

3. Guilleminault C, Palombini L, Poyares D, Chowdhuri S. Chronic insomnia, postmenopausal women, and sleep disordered breathing: part 1. Frequency of sleep disordered breathing in a cohort. J Psychosom Res 2002;53:611-615.

4. Krakow B, Melendrez D, Ferreira E, et al. Prevalence of insomnia symptoms in patients with sleep-disordered breathing. Chest 2001;120: 1923-1929.

5. Krakow B, Melendrez D, Pedersen B, et al. Complex insomnia: insomnia and sleep-disordered breathing in a consecutive series of crime victims with nightmares and PTSD. Biol Psychiatry 2001;49:948-953.

6. Guo D, Peng CK, Wu HL, et al. ECG-derived cardiopulmonary analysis of pediatric sleep-disordered breathing. Sleep Med 2011;12:384-389.

7. Harrington J, Schramm PJ, Davies CR, Lee-Chiong TL Jr. An electrocardiogram-based analysis evaluating sleep quality in patients with obstructive sleep apnea. Sleep Breath 2013;17:1071-1078.

8. Schramm PJ, Thomas R, Feige B, Spiegelhalder K, Riemann D. Quantitative measurement of sleep quality using cardiopulmonary coupling analysis: a retrospective comparison of individuals with and without primary insomnia. Sleep Breath 2013;17:713-721.

9. Rechtschaffen A, Kales A. A manual of standardized terminology, techniques and scoring systems for sleep stages of human subjects. Washington, DC: Brain Information Service/Brain Research Institute, University of California, 1968.

10. Iber C, Ancoli-Israel S, Chesson AL, Quan SF. The AASM Manual for the scoring of sleep and associated events: rules, terminology and technical specifications. Westchester, IL: American Academy of Sleep Medicine, 2007.

11. American Sleep Disorders Association. EEG arousals: scoring rules and examples: a preliminary report from the Sleep Disorders Atlas Task Force of the American Sleep Disorders Association. Sleep 1992;15:173-184.

12. Thomas RJ, Mietus JE, Peng CK, et al. Differentiating obstructive from central and complex sleep apnea using an automated electrocardiogram-based method. Sleep 2007;30:1756-1769.

13. Thomas RJ, Mietus JE, Peng CK, Goldberger AL. An electrocardiogrambased technique to assess cardiopulmonary coupling during sleep. Sleep 2005;28:1151-1161.

14. Chung KF. Relationships between insomnia and sleep-disordered breathing. Chest 2003;123:310-311; author reply 311-313.

15. Lavie P, Pillar G. Gender and age differences in symptoms' profile insleep apnea syndrome: a possible cause of gender bias in diagnosis. Somnologie 2001;5:93-96.

16. Quintana-Gallego E, Carmona-Bernal C, Capote F, et al. Gender differences in obstructive sleep apnea syndrome: a clinical study of 1166 patients. Respir Med 2004;98:984-989.

17. Gooneratne NS, Gehrman PR, Nkwuo JE, et al. Consequences of comorbid insomnia symptoms and sleep-related breathing disorder in elderly subjects. Arch Intern Med 2006;166:1732-1738.

18. Lichstein KL, Riedel BW, Lester KW, Aguillard RN. Occult sleep apnea in a recruited sample of older adults with insomnia. J Consult Clin Psychol 1999;67:405-410.

19. Roehrs T, Conway W, Wittig R, Zorick F, Sicklesteel J, Roth T. Sleepwake complaints in patients with sleep-related respiratory disturbances. Am Rev Respir Dis 1985;132:520-523.

20. Backhaus J, Junghanns K, Broocks A, Riemann D, Hohagen F. Test-retest reliability and validity of the Pittsburgh Sleep Quality Index in primary insomnia. J Psychosom Res 2002;53:737-740.

21. Krell SB, Kapur VK. Insomnia complaints in patients evaluated for obstructive sleep apnea. Sleep Breath 2005;9:104-110.

22. Smith S, Sullivan K, Hopkins W, Douglas J. Frequency of insomnia report in patients with obstructive sleep apnoea hypopnea syndrome (OSAHS). Sleep Med 2004;5:449-456. 